\title{
A case study of dayside reconnection under extremely low solar wind density conditions
}

\author{
R. Maggiolo ${ }^{1}$, J. A. Sauvaud ${ }^{2}$, I. Dandouras ${ }^{2}$, E. Luceck ${ }^{3}$, and H. Rème ${ }^{2}$ \\ ${ }^{1}$ IASB, 3 Avenue Circulaire, Bruxelles, Belgium \\ ${ }^{2}$ CESR, 9 Avenue du Colonel Roche, Toulouse 31028, France \\ ${ }^{3}$ Imperial College London, South Kensington Campus, London SW7 2AZ, UK \\ Received: 30 May 2008 - Revised: 9 October 2008 - Accepted: 17 October 2008 - Published: 17 November 2008
}

\begin{abstract}
From 15 February 2004, 20:00 UT to 18 February 2004, 01:00 UT, the solar wind density dropped to extremely low values (about $0.35 \mathrm{~cm}^{-3}$ ). On 17 February, between 17:45 UT and 18:10 UT, the CLUSTER spacecraft cross the dayside magnetopause several times at a large radial distance of about $16 R_{E}$. During each of these crossings, the spacecraft detect high speed plasma jets in the dayside magnetopause and boundary layer. These observations are made during a period of southward and dawnward Interplanetary Magnetic Field (IMF). The magnetic shear across the local magnetopause is $\sim 90^{\circ}$ and the magnetosheath beta is very low $(\sim 0.15)$.
\end{abstract}

We evidence the presence of a magnetic field of a few $\mathrm{nT}$ along the magnetopause normal. We also show that the plasma jets, accelerated up to $600 \mathrm{~km} / \mathrm{s}$, satisfy the tangential stress balance. These findings strongly suggest that the accelerated jets are due to magnetic reconnection between interplanetary and terrestrial magnetic field lines northward of the satellites. This is confirmed by the analysis of the ion distribution function that exhibits the presence of $\mathrm{D}$ shaped distributions and of a reflected ion population as predicted by theory. A quantitative analysis of the reflected ion population reveals that the reconnection process lasts about $30 \mathrm{~min}$ in a reconnection site located at a very large distance of several tens $R_{E}$ from the Cluster spacecraft. We also estimate the magnetopause motion and thickness during this event.

This paper gives the first experimental study of magnetic reconnection during such rare periods of very low solar wind density. The results are discussed in the frame of magnetospheric response to extremely low solar wind density conditions.

Correspondence to: R. Maggiolo

(maggiolo@aeronomie.be)
Keywords. Magnetospheric physics (Magnetopause, cusp, and boundary layers; Solar wind-magnetosphere interactions)

\section{Introduction}

The solar wind consists of a continuous flow of charged particles reaching the earth with an average density of $10 \mathrm{~cm}^{-3}$ and an average velocity of about $450 \mathrm{~km} / \mathrm{s}$. The solar wind plasma consists primarily of hot electrons and protons with a minor fraction of $\mathrm{He}^{2+}$ ions and some other heavier ions. However, the solar wind is dynamic and can vary strongly. Typically, its velocity can vary from $200 \mathrm{~km} / \mathrm{s}$ to $900 \mathrm{~km} / \mathrm{s}$, its density between $0.3 \mathrm{~cm}^{-3}$ and $100 \mathrm{~cm}^{-3}$ and the fraction of $\mathrm{He}^{2+}$ from 0 to 25 percent. The solar wind pressure, defined as $P_{D}=\rho V^{2}$ where $\rho$ is the solar wind mass density and $V$ the solar wind velocity, controls the size of the magnetosphere. It is also highly variable and reaches its lower values during periods of extremely low solar wind density. Such periods are very rare and typically last between $12 \mathrm{~h}$ and $24 \mathrm{~h}$. ACE data reveal that from 1 January 1996 to 31 March 2005, the solar wind dynamic pressure was lower than $0.2 \mathrm{nPa}$ only during $267 \mathrm{~h}$. They provide a good opportunity to examine solar-terrestrial interactions when the solar wind pressure on the magnetosphere is extremely low. A recent study of Janardhan et al. (2008) identified the source region of the low density solar wind for two extremely low solar wind density periods as being active regions complexes located at the sun central meridian. They also showed that they were characterized by highly nonradial solar wind outflows.

One event in particular, taken on 11 May 1999 and known to the community as "the day the solar wind disappeared", has been widely studied in a special issue of Geophysical

Published by Copernicus Publications on behalf of the European Geosciences Union. 


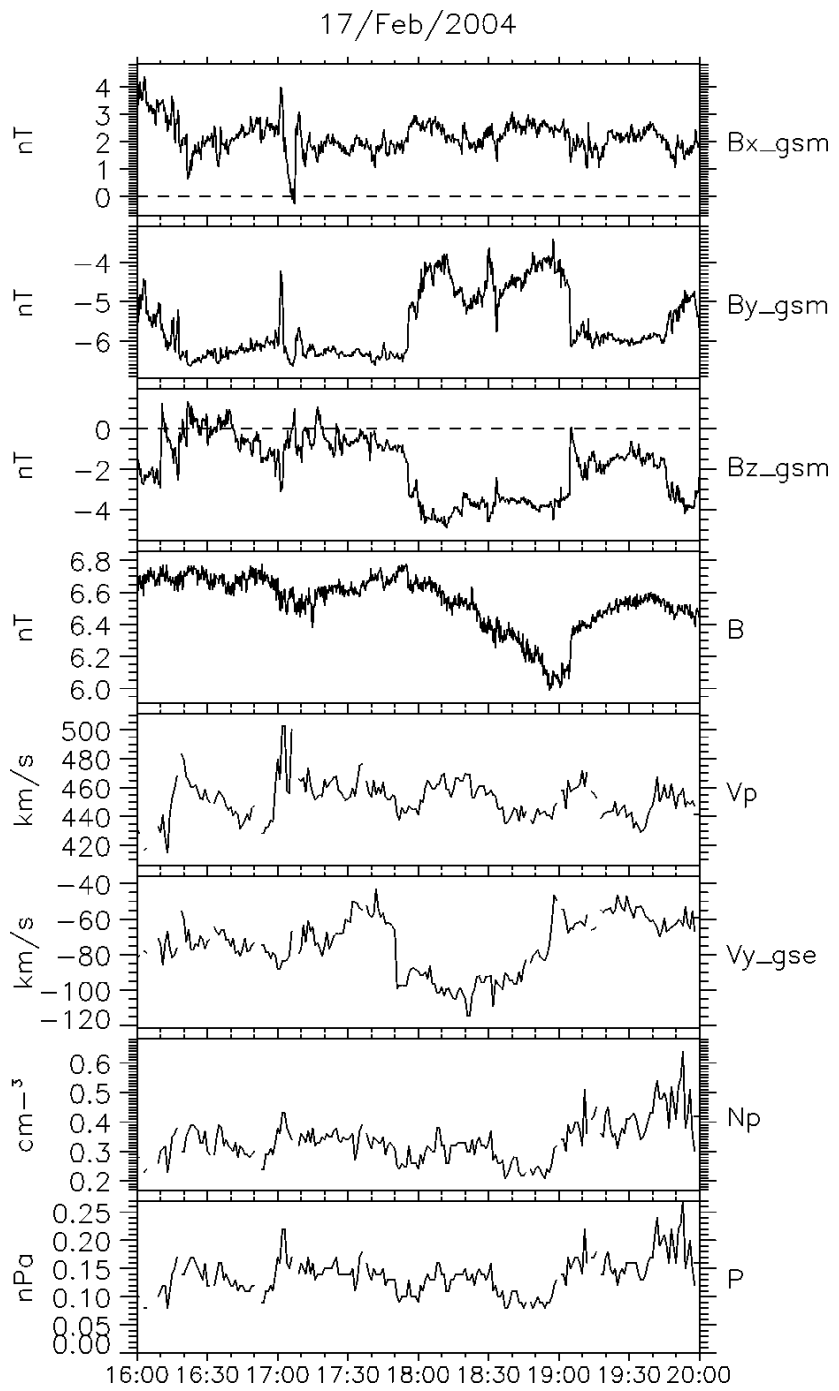

Fig. 1. 17 February 2004. From top to bottom: $B_{X}, B_{Y}$ and $B_{Z}$ IMF components in the GSM frame as measured by ACE.

Magnetic field magnitude as measured by ACE.

Solar wind velocity magnitude and Y component in he GSE frame as measured by ACE.

Density and dynamic pressure as measured by ACE.

The data have been shifted by $49 \mathrm{~min}$ to account for the propagation time from ACE to the Cluster spacecraft.

Research Letters (Tenuous Solar Wind, 2000). Studies performed during the 11 May event revealed several striking features. The magnetosphere had grown to exceptionally large dimensions due to the low solar wind pressure, about 100 times larger in volume than during average solar wind conditions (Lockwood et al., 2001). While the solar wind energy and density input to the magnetosphere was very low, Parks et al. (2000) showed that the auroral oval was still active and that the auroral activity was rather linked to the orientation of IMF $B_{Z}$ than to the solar wind density. Furthermore, Terasawa et al. (2000) evidenced using Geotail data that the plas- masheet and the magnetotail surprisingly maintained during this extremely low solar wind density period.

In this study, we will discuss a period when the solar wind density dropped to about $5 \%$ of its average value leading to a very low solar wind dynamic pressure $(\sim 0.15 \mathrm{nPa})$. During this event the Cluster spacecraft detect several high speed plasma jets at the inner edge of the magnetopause.

The presence of high speed plasma flows in this region of the magnetosphere is known to be a possible signature of magnetic reconnection between solar and magnetospheric magnetic field lines. Convincing experimental evidence has been given by in-situ observations in the magnetopause from the ISEE and AMPTE satellites (e.g. Paschmann et al., 1979; Sonnerup et al., 1981) to the most recent missions like Cluster (e.g. Bosqued et al., 2001; Bavassano-Cattaneo, 2006). Evidence for magnetic reconnection can be obtained both by fluid and kinetic considerations. They can give complementary and independent information about the occurrence of magnetic reconnection. The fluid evidence consists of testing the tangential stress balance across the magnetopause. This test is named the Walén test (Hudson, 1970; Paschmann et al., 1979; Sonnerup et al., 1981). Kinetic evidence is given by the observation in the particle distribution function of transmitted magnetosheath ions with a D-shaped distribution function and of reflected magnetosheath ions (Cowley, 1982).

In this paper, we will study the magnetopause and plasma jets properties in the frame of magnetic reconnection and discuss the results in term of magnetospheric response to the extremely low solar wind density. We first start with a brief presentation of the instrumentation used for this study. Then, we describe the observations. In Sect. 3, we discuss the fluid properties of the magnetopause to evidence the robustness of magnetic reconnection. In Sect. 4, we study the ion distribution function. It confirms the occurrence of magnetic reconnection and allows us to estimate the location of the reconnection site. Section 5 is dedicated to the study of the magnetopause motion and thickness. Section 6 summarizes the results and interpretations.

\section{Instrumentation}

The four identical Cluster satellites have been launched in 2000 on an elliptical orbit $\left(4.0 \times 19.6 R_{E}\right)$ with an inclination of $90^{\circ}$. A detailed description of the Cluster mission can be found in the paper of Escoubet et al. (2001).

We use data from the Cluster Ion Spectrometers (CIS) onboard the Cluster spacecraft 1, 3 and 4. The CIS instrument is described in detail in Rème et al. (2001). It consists of two different ion spectrometers, the Composition and Distribution Function analyser (CODIF), which can resolve the major magnetospheric ions, and the Hot Ion Analyzer (HIA), which has no mass resolution but higher angular and energy resolutions. 


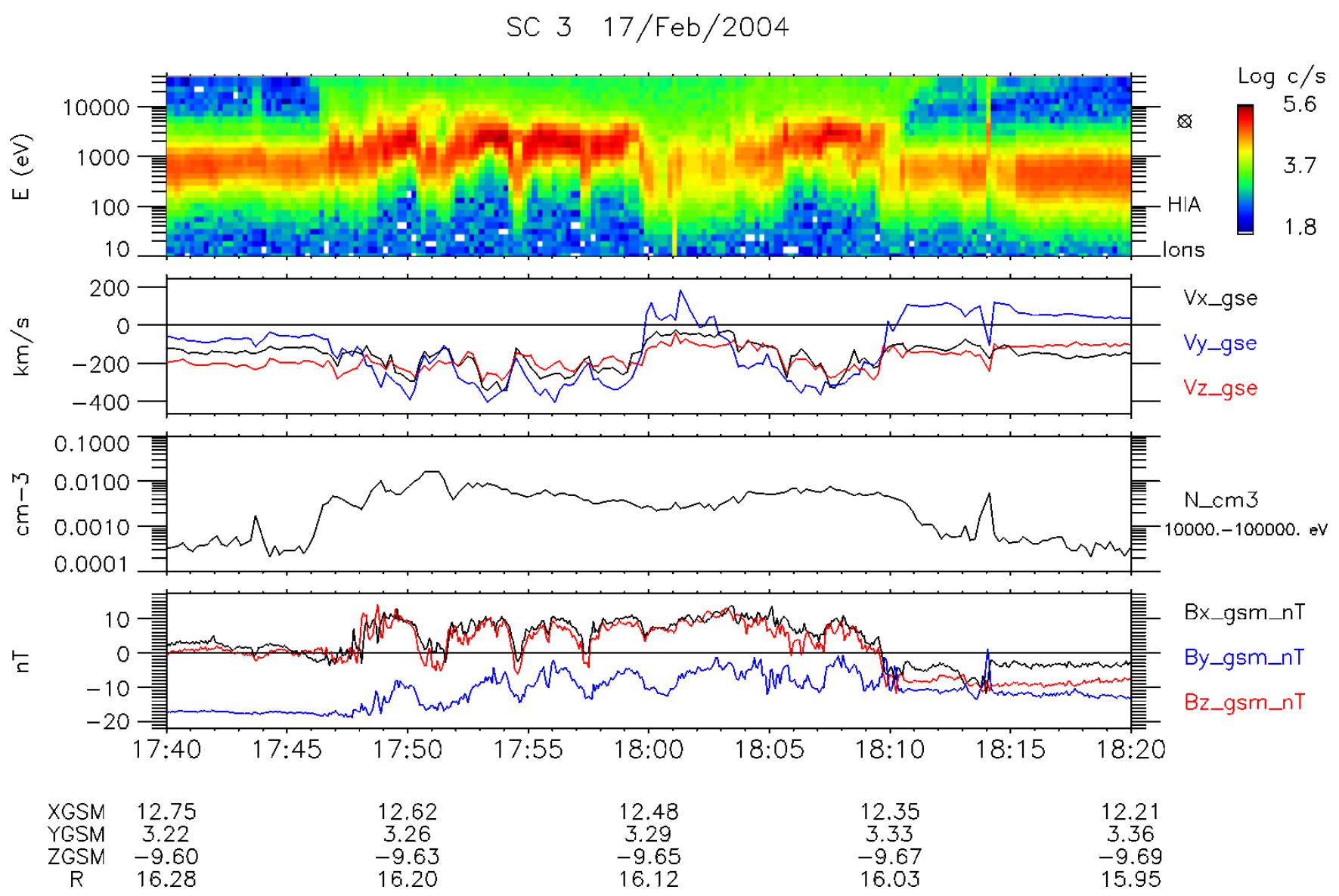

Fig. 2. Magnetic field and ion data from Cluster spacecraft 3 from 17:35 UT to 18:30 UT. From top to bottom: Energy-time spectrogram from HIA for all ions.

Ion velocity in the GSE frame computed from HIA.

Partial ion density for particles with energies higher than $10 \mathrm{keV}$ computed from HIA.

Magnetic filed components in the GSM frame computed from FGM.

In addition, we use high-resolution $(5 \mathrm{~Hz})$ magnetic field data from the Cluster Fluxgate Magnetometers (FGM) (Balogh et al., 2001).

The ACE data are used to monitor the solar wind conditions. These data have been shifted in time to account for the solar wind propagation from ACE to the Cluster spacecraft.

\section{Observations}

From 15 February 2004, 20:00 UT to 18 February 2004, 01:00 UT, the solar wind density dropped to extremely low values $\left(\sim 0.35 \mathrm{~cm}^{-3}\right)$. In this study, we will focus on a time period taken on 17 February 2004 from 17:50 UT to 18:15 UT.

The Cluster satellites are located approximately in the $(X Z)_{\mathrm{GSE}}$ plane in the Southern Hemisphere of the dayside magnetosheath (SC4, 18:00 UT: $X_{\mathrm{GSE}}=12.49 R_{E}$, $\left.Y_{\mathrm{GSE}}=-0.72 R_{E}, Z_{\mathrm{GSE}}=-10.19 R_{E}\right)$ at a geocentric distance of $16.14 R_{E}$. The separation distance between the Cluster spacecraft is around $500 \mathrm{~km}$. During the considered time period the Cluster spacecraft are in the magnetosheath skimming the magnetopause. They are travelling in the direction of the magnetosphere where they enter after 21:00 UT.

The IMF, the solar wind density and velocity as given by the ACE spacecraft from 16:00 UT to 20:00 UT are presented in Fig. 1. These data have been time shifted by $49 \mathrm{~min}$ to account for the solar wind propagation from ACE to Cluster. Before 19:00 UT the solar wind density is about $0.35 \mathrm{~cm}^{-3}$ and the dynamic pressure around $0.12 \mathrm{nPa}$. Then they slightly increase to reach respectively $0.5 \mathrm{~cm}^{-3}$ and $0.17 \mathrm{nPa}$. The solar wind velocity, about $450 \mathrm{~km} / \mathrm{s}$, is almost constant during this time period. Due to its low density, the solar wind Alfvén velocity is high $(\sim 250 \mathrm{~km} / \mathrm{s})$ and the solar wind is only weakly super Alfvénic $\left(n_{A} \sim 1.8\right)$. It is interesting to notice that during this event, as noted by Janardhan et al. (2008) for other periods of low solar wind density, the solar wind 

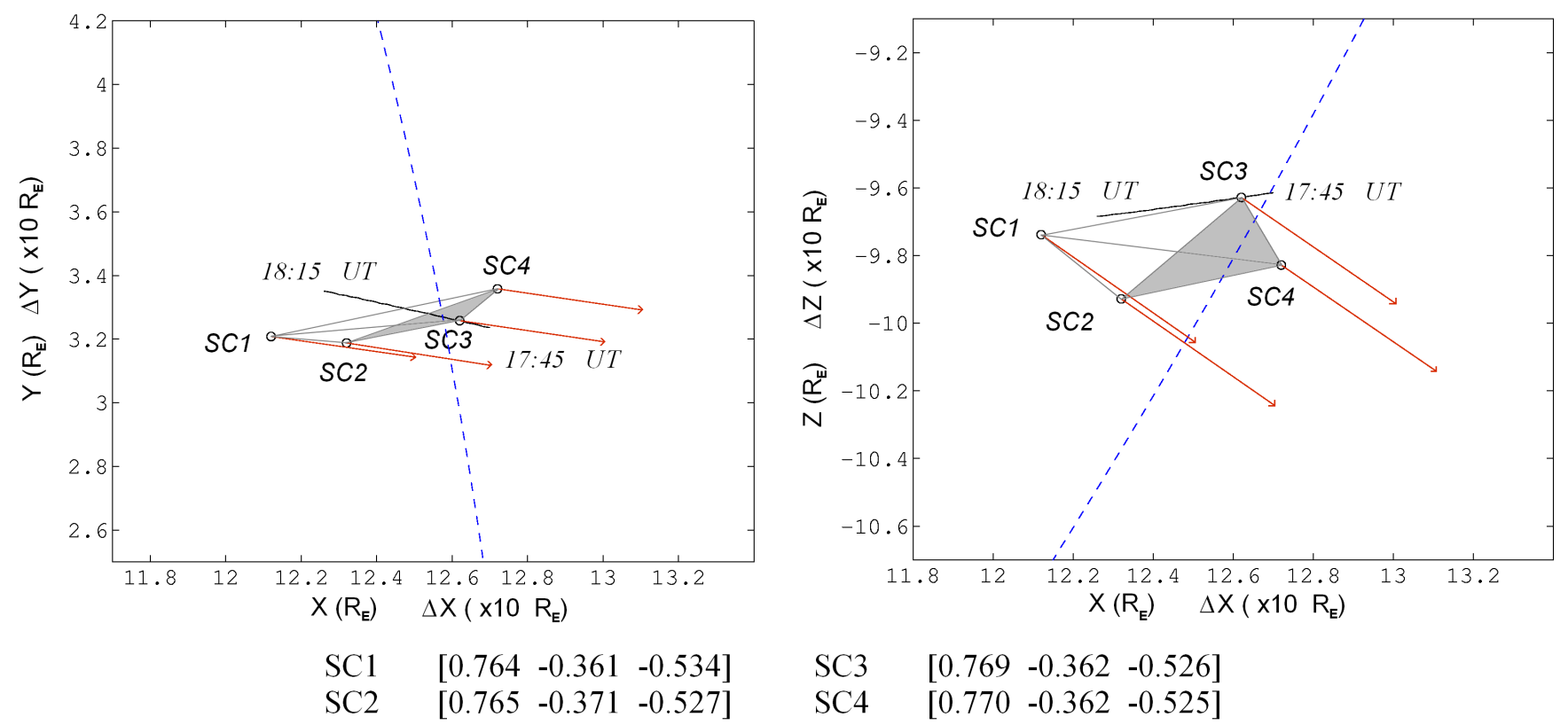

Fig. 3. Cluster spacecraft 3 orbit on 17 February 2004 from 17:45 UT to 18:15 UT projected in the X-Y (left) and X-Z (right) GSM planes (black line). The Cluster tetrahedron at 17:50 UT is also plotted. The separation distance between spacecraft has been multiplied by a factor of 10. The red arrows represent the components of the magnetopause normal predicted by the minimum variance analysis applied on magnetic field for each spacecraft. The blue dotted curve represents the magnetopause location as predicted by the Shue et al. (1997) model.

deviates from the sun-earth direction. According to ACE data, the $Y_{\mathrm{GSE}}$ component of the solar wind velocity reaches $-100 \mathrm{~km} / \mathrm{s}$. Just before 18:00 UT, the IMF turns from a dawnward orientation $\left(B_{X \mathrm{GSM}} \sim 2 \mathrm{nT}, B_{\mathrm{YGSM}} \sim-6.5 \mathrm{nT}\right.$, $\left.B_{Z \mathrm{GSM}} \sim-0.5 \mathrm{nT}\right)$ to a dawnward/southward orientation $\left(B_{X \mathrm{GSM}} \sim 2.5 \mathrm{nT}, B_{Y \mathrm{GSM}} \sim-4 \mathrm{nT}, B_{Z \mathrm{GSM}} \sim-4 \mathrm{nT}\right)$ with almost no variations of $B_{X \mathrm{GSM}}$. This turning is accompanied by a slow decrease of the total magnetic field strength from about 6.8 to $6.2 \mathrm{nT}$.

Cluster data from 17:40 UT to 18:20 UT are presented in Fig. 2. Until 17:46 UT, the Cluster spacecraft are located in the magnetosheath. The magnetic field measured by the FGM instrument is mainly dawnward directed $\left(B_{X \mathrm{GSM}} \sim 2 \mathrm{nT}, B_{Y \mathrm{GSM}} \sim-17 \mathrm{nT}, B_{Z \mathrm{GSM}} \sim 0 \mathrm{nT}\right)$ in a direction consistent with the IMF orientation. The plasma has characteristic magnetosheath temperature $(T i \sim 500 \mathrm{eV})$ and the ion velocity is mainly oriented in the southward/tailward direction as expected for magnetosheath plasma flowing around the magnetopause. However, the density $\left(1 \mathrm{~cm}^{-3}\right)$ is well below the usual magnetosheath density $\left(\sim 25 \mathrm{~cm}^{-3}\right)$ and the magnetosheath plasma $\beta$ is very low $(0.15)$.

From 17:47 UT to $18: 10 \mathrm{UT}$, a succession of magnetic field turnings is detected. $B_{Y G S M}$ becomes closer to $0 \mathrm{nT}$ and $B_{X \mathrm{GSM}}$ and $B_{Z \mathrm{ZSM}}$ reach similar values around $10 \mathrm{nT}$. This orientation is consistent with the expected magnetospheric field lines orientation at the Cluster location. These successive magnetic field rotations prove unambiguously that the Cluster spacecraft cross the magnetopause several times during this time period. According to the magnetic field variations, Cluster magnetopause crossings occur quasi periodically with a period of about 3-4 min. These periodic crossings are caused by large-scale oscillations of the magnetopause probably triggered by solar wind pressure fluctuations (e.g. Sibeck et al., 1991). Due to the low separation distance between Cluster spacecraft, no information can be obtained on the propagation and wavelength of these oscillations. We just can state that the amplitude of these oscillations is higher than the current layer thickness and that they are associated with a magnetopause motion at a velocity of about $20 \mathrm{~km} / \mathrm{s}$ along the magnetopause normal (see Sect. 5). Simultaneously, the CIS experiment detects high-energy particles $(>10 \mathrm{keV})$ with a large amount of $\mathrm{O}^{+}$ions, presumably of magnetospheric origin. These magnetospheric ions are also observed in the magnetosheath close to the magnetopause. Each of the magnetopause crossings is associated with the detection of high speed ion beams with velocities reaching $600 \mathrm{~km} / \mathrm{s}$. The flow speed enhancement across the magnetopause relative to the magnetosheath flow is higher than $300 \mathrm{~km} / \mathrm{s}$ and accelerated plasma jets are oriented dawnward/southward/tailward. The peak of the flow speed always occurs at the magnetospheric side of the current layer.

After 18:10 UT the Cluster spacecraft return in the magnetosheath where the magnetic field is now oriented southward $\left(B_{Z G S M} \sim-9 \mathrm{nT}\right)$. It is therefore clear that the southward 
IMF turning detected by ACE actually reached the Cluster spacecraft between 17:50 UT and 18:10 UT. However, it may have reached others regions of the dayside magnetopause before Cluster observation of the $B_{Z}$ turning. This magnetic field reversal created favourable conditions for magnetic reconnection at the dayside magnetopause.

\section{Evidence for magnetic reconnection}

In this section, we discuss the properties magnetopause and of the accelerated ion beams to show fluid and kinetic evidence for magnetic reconnection.

\subsection{Magnetopause orientation}

As a first step, we performed a variance analysis on magnetic field data for each Cluster spacecraft respectively for the full time interval (17:47 UT to $18: 11$ UT) and for each individual crossing to determine the magnetopause orientation. The normal orientation obtained for each spacecraft and for each time interval is very similar with differences of a few degrees, not meaningful as it is comparable with the uncertainty associated with this method.

Figure 3 presents a projection of the magnetopause normal vector obtained by the minimum variance analysis for the full time interval respectively in the $(X Y)_{\mathrm{GSM}}$ and $(X Z)_{\mathrm{GSM}}$ planes. The location and orientation of the magnetopause are compared with an interpolation of the Shue et al. (1997) magnetopause model. Whereas this model is defined for solar wind dynamic pressures higher than $0.5 \mathrm{nPa}$, we interpolated it for a dynamic pressure of $0.15 \mathrm{nPa}$. Both magnetopause location and orientation obtained with this model are in good agreement with Cluster data. However, the magnetopause orientation in the $(X Y)_{\text {GSE }}$ plane obtained by the minimum variance analysis on Cluster magnetic field data slightly differs with the magnetopause orientation given by the Shue et al. model. This is possibly an effect of the azimuthal component of the solar wind $\left(V_{Y \mathrm{GSE}} \sim-100 \mathrm{~km} / \mathrm{s}\right)$ that slightly distort the magnetopause, shifting its nose in the $+Y_{\mathrm{GSE}}$ direction. Note that, according to the Shue et al. (1997) magnetopause model, the magnetopause extends at a radial distance of about $16 R_{E}$ at the subsolar point.

Then we projected the magnetic field on the magnetopause frame, using the magnetopause orientation given by the minimum variance analysis. This is exemplified in Fig. 4 which presents the hodogram obtained with spacecraft 3 magnetic field data for the whole time interval, from 17:47 UT to 18:11 UT. It clearly evidences a rotation of about $90^{\circ}$ of the magnetic field in the magnetopause plane for each magnetopause crossing (left panel) and the presence of a magnetic field along the magnetopause normal (right panel). The eigenvalues $\lambda_{1}, \lambda_{2}$ and $\lambda_{3}$ of the magnetic variance matrix are also indicated. They fulfil quite satisfactorily the condi-

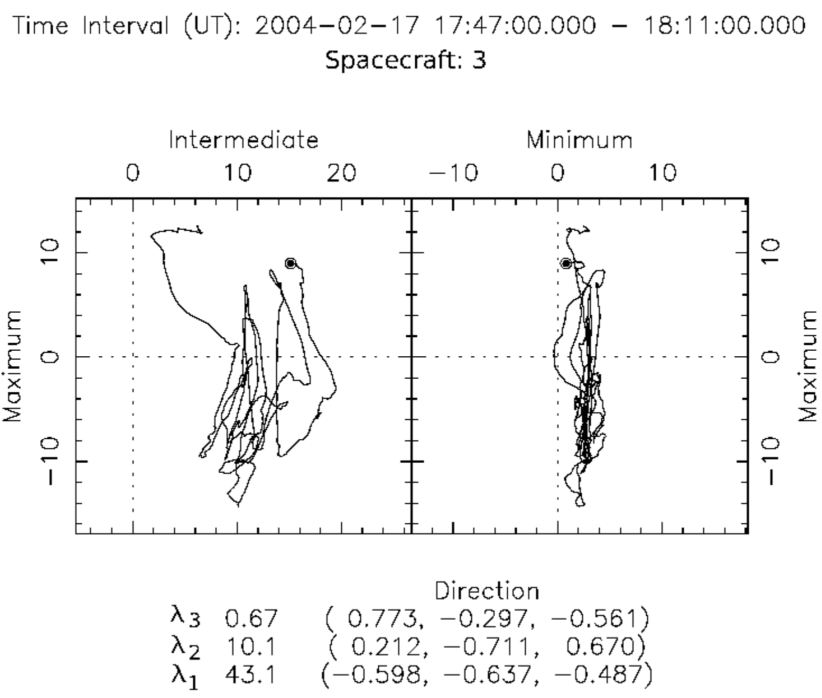

Fig. 4. Magnetic field hodogram obtained by minimum variance analysis applied on Cluster 3 magnetic field data between 17:47:00 UT and 18:11:00 UT. The left pane represents the projection of the magnetic field in the plane containing the maximum and intermediate variance axis, i.e. in the magnetopause plane. The left panel represents the projection of the magnetic field in the plane containing the minimum and maximum variance axis. The components in the GSE a plane of respectively the minimum, intermediate and maximum variance axis are given in the bottom of this figure.

tion $\lambda_{1}, \lambda_{2} \gg \lambda_{3}$ required to have a reliable estimation of the magnetopause normal.

The presence of normal magnetic field, comprised between 1-2 nT and directed toward the exterior of the magnetosphere is clearly visible. A similar analysis by the others Cluster spacecraft gives comparable results. A non-zero magnetic field along the magnetopause normal indicates that the magnetopause consists of a rotational discontinuity during this event as expected when magnetic reconnection occurs. Because the normal magnetic field is oriented toward the exterior of the magnetosphere, if magnetic reconnection occurs, the reconnection site is located northward of the spacecraft. The magnitude of the normal magnetic field is difficult to estimate accurately because of statistical errors and physical approximations (one-dimensional discontinuity and time stationarity). The first one is inversely proportional to $M^{1 / 2}$, where $M$ is the number of data points used for the minimum variance analysis. This error is important when the analysis is made for a single crossing as the data set is relatively small. If the analysis is made for the full time interval the statistical error fall but the uncertainty due to physical approximations increases because adjoining structures are included and because the time stationarity hypothesis is less satisfied for a long time period. Furthermore, it doesn't take into account any change of the normal orientation from one crossing to another. The uncertainty on the determination of the normal magnetic field magnitude is difficult to estimate, 


\section{$17 /$ Feb $/ 2004$}
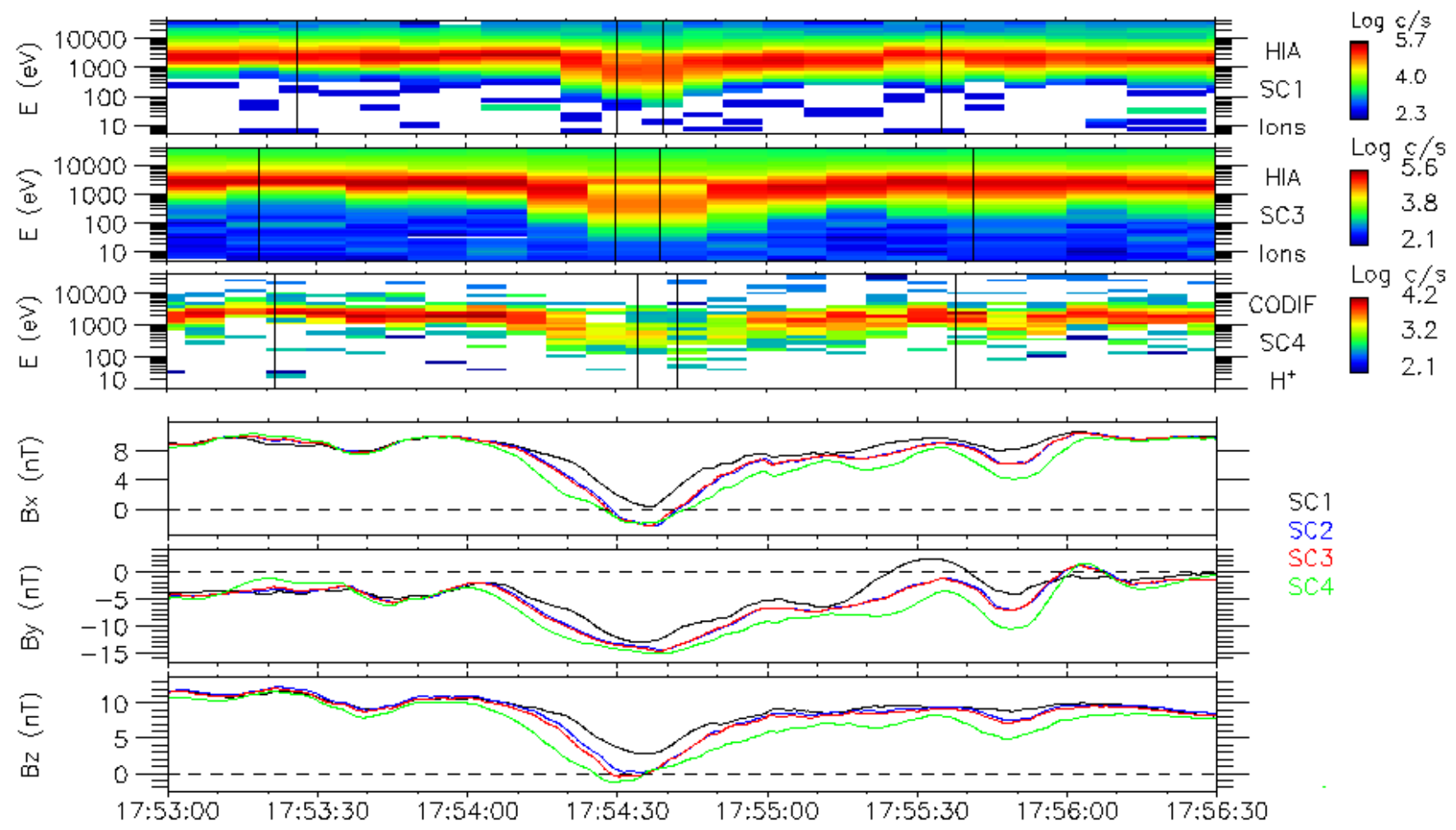

Fig. 5. 17 February 2004 from 17:53 UT to 17:56:30 UT. From top to bottom:

Energy-time spectrogram from HIA for all ions, spacecraft 1.

Energy-time spectrogram from HIA for all ions, spacecraft 3.

Energy-time spectrogram from CODIF for $\mathrm{O}^{+}$ions, spacecraft 4.

Magnetic field $B_{X}$ component in the GSE frame for the 4 Cluster spacecraft for Cluster 1 (black), Cluster 2 (blue), Cluster 3 (red) and Cluster 4 (green) computed from FGM data. Magnetic field $B_{Y}$ component in the GSE frame for the 4 Cluster spacecraft with the same colour code computed from FGM data. Magnetic field $B_{Z}$ component in the GSE frame for the 4 Cluster with the same colour code spacecraft computed from FGM data.

however the Fig. 4 evidences that we do not obtain an accurate result.

Furthermore it must be stressed that, although low $\left(\ll 180^{\circ}\right)$, the local magnetic shear can not be used to evaluate the validity of component or anti-parallel reconnection as the reconnection site may be far from the Cluster spacecraft (see below).

\subsection{DeHoffmann-Teller analysis and Walén test}

In this part, we discuss in more detail two consecutive magnetopause crossings, chosen arbitrarily, respectively from the magnetosphere to the magnetosheath and from the magnetosheath to the magnetosphere. The results obtained for the other magnetopause crossings are similar. Figure 5 presents Cluster data during these two crossings. Cluster spacecraft are first located in the magnetospheric boundary layer characterized by the presence of accelerated ion beams and by a magnetic field with a magnetospheric orientation. Around
17:54:30 UT the spacecraft enter briefly the magnetosheath and then return to the boundary layer.

Magnetohydrodynamic models for 1-dimensional steady state reconnection predict that the flow is Alfvénic in the deHoffmann-Teller (dHT) frame of reference (Hudson, 1970). We will use the tangential stress balance to examine the occurrence of Alfvénic flow acceleration. The time intervals used to check the tangential stress balance for each spacecraft are indicated by vertical lines in Fig. 5 .

The first step consists to search for the existence of a welldefined dHT frame for each spacecraft. In this frame, moving with a velocity $V_{H T}$ along the presumed rotational discontinuity, the electric field vanishes. To obtain this frame we will use the method described in detail by Khrabrov and Sonnerup (1998). The $V_{H T}$ velocity is obtained by minimizing the quantity:

$D=\sum_{m}\left|\left(\boldsymbol{V}^{m}-\boldsymbol{V}_{H T}\right) \times \boldsymbol{B}^{m}\right|^{2}$ 
Table 1. dHT analysis results for the two consecutive magnetopause crossings discussed in detail in this section. Velocities are in $\mathrm{km} / \mathrm{s}$ and acceleration in $\mathrm{km} / \mathrm{s}^{2}$. All vectors are expressed in the GSE frame. The results presented here have been obtained by introducing a constant acceleration of the dHT frame: $\boldsymbol{V}_{H T}=\boldsymbol{V}_{H T 0}+\boldsymbol{a}_{H T} t . p_{H T}$ is the fitting slope between $\boldsymbol{E}_{H T}=-\boldsymbol{V}_{H T} \times \boldsymbol{B}$ and $\boldsymbol{E}_{C}=-\boldsymbol{V} \times \boldsymbol{B}$, the convection electric field. $D / D_{0}$ is the least square quality factor. $V_{H T \text { av }}$ is the average velocity of the dHT frame.

\begin{tabular}{|c|c|c|c|c|}
\hline $\boldsymbol{V}_{H T 0}$ & $\boldsymbol{V}_{H T \text { av }}$ & $a_{H T}$ & $p_{H T}$ & $D / D_{0}$ \\
\hline$(-214.02-446.84-131.23)$ & $\begin{array}{r}\text { SC } 1 \text { 17:53:26-17:54:31 (N } \\
(-186.27-442.29-129.57) \\
17: 54: 39-17: 55: 35(\mathrm{Msp}\end{array}$ & $\begin{array}{l}\text { Msh-Msph) } \\
(0.8540 .1400 .051) \\
\text { ph-Msh) }\end{array}$ & 1.0001 & 0.00125 \\
\hline$(-169.11-328.77-154.18)$ & $(-201.37-379.81-144.13)$ & $(-1.152-1.8230 .359)$ & 1.0002 & 0.00397 \\
\hline$(-324.17-405.03-239.31)$ & $\begin{array}{r}\text { SC } 3 \text { 17:53:18-17:54:30 (N) } \\
(-216.57-366.83-179.51) \\
17: 54: 54-17: 55: 42(\mathrm{Msp}\end{array}$ & $\begin{array}{l}\text { Msh-Msph) } \\
(2.9891 .0611 .661) \\
\text { ph-Msh) }\end{array}$ & 1.0003 & 0.00830 \\
\hline$(-143.46-307.06-135.25)$ & $(-171.66-341.13-139.02)$ & $(-1.175-1.427-0.157)$ & 0.9999 & 0.00777 \\
\hline$(-163.31-403.07-106.72)$ & $\begin{array}{c}\text { SC } 4 \text { 17:53:22-17:54:34 (N } \\
(-174.36-346.94-133.75) \\
\text { 17:54:42-17:55:38 (Msp }\end{array}$ & $\begin{array}{l}\text { Msh-Msph) } \\
(-0.3701 .559-0.751) \\
\text { ph-Msh) }\end{array}$ & 1.0000 & 0.01247 \\
\hline$(-150.00-208.26-93.30)$ & $(-181.19-272.82-132.28)$ & $(-1.114-2.305-1.392)$ & 1.0001 & 0.01266 \\
\hline
\end{tabular}

using the measured magnetic field $\left(\boldsymbol{B}^{m}\right)$ and ion velocity $\left(\boldsymbol{V}^{m}\right)$ during the discontinuity crossing. This method can be improved by choosing a dHT frame continuously accelerated $\left(\boldsymbol{V}_{H T}=\boldsymbol{V}_{H T 0}+\boldsymbol{a}_{H T} t\right)$ instead of a dHT frame moving at a constant velocity.

Both methods have been used. The results are comparable although the dHT frame velocities obtained with the accelerated frame are more similar from one spacecraft to another (Table 1). There is a very strong correlation between $\boldsymbol{E}_{H T}=-\boldsymbol{V}_{H T} \times \boldsymbol{B}$ and the convection electric field, with fitting slopes very close to 1 and very small least-squares residuals. We can thus conclude that for each spacecraft a welldefined dHT frame exists. This frame is mainly moving along the magnetopause at an average velocity of $430 \mathrm{~km} / \mathrm{s}$ in the dawnward/southward/tailward direction. Its velocity along the magnetopause normal (about tens of $\mathrm{km} / \mathrm{s}$ ) corresponds to the magnetopause motion along its normal and will be discussed later on.

The second step is the tangential stress balance or Walén test (Hudson, 1970; Paschmann et al., 1979; Sonnerup et al., 1981). It consists of checking if the plasma is Alfvénic in the dHT frame across the discontinuity, i.e. if:

$\boldsymbol{V}-\boldsymbol{V}_{H T}= \pm \boldsymbol{V}_{A}$

The sign + is for a reconnection site located southward and the sign - for a reconnection site located northward. $V$ is the plasma velocity, $\boldsymbol{V}_{H T}$ the dHT frame velocity and $\boldsymbol{V}_{A}$ the Alfvén velocity given by:

$\boldsymbol{V}_{A}=\boldsymbol{B}\left(\frac{1-\alpha}{\mu_{0} \rho}\right)^{1 / 2}$ where

$\alpha=\left(\rho_{/ /}-\rho_{\perp}\right) \frac{\mu_{0}}{B^{2}}$

is the anisotropy factor.

The results of this test, given in Table 2, present the fitting slopes between the plasma velocity in the dHT frame and the Alfvén velocity obtained using a component-by-component scatter plot of these velocities. The corresponding correlation coefficient is also indicated. These two velocities are well correlated but the plasma velocity in the dHT frame is only 46 to 62 percent of the Alfvén velocity.

This is certainly a consequence of the inadequate operating mode of the CIS experiment during this time period. Indeed, due to the large geocentric distance of Cluster spacecraft, the CIS experiment is operating in a mode adapted to solar wind measurement. The consequence is that the ion distribution function sampling is incomplete. It causes an underestimation of a factor of about 2 of the ion density with no significant error on the determination of the ion velocity. In solar wind mode, the high sensitivity face of CODIF and HIA instruments doesn't detect particles with energies lower than $2.5 \mathrm{keV}$ for the angular sectors directed toward the sun. The error in density measurement depends on the proportion of the ion population located in those sectors and thus of the arrival direction of ions on the detector. The error in the magnetosheath region can be estimated when the instrument changes operating mode at around 18:15 UT when Cluster spacecraft are located in the magnetosheath. At that time the ion density increases from about 0.3 to $0.7 \mathrm{~cm}^{-3}$. It implies that the density is underestimated by a factor of about 2 . The error in velocity determination is less important as the 
CIS HIA $17 / \mathrm{Fev} / 2004$

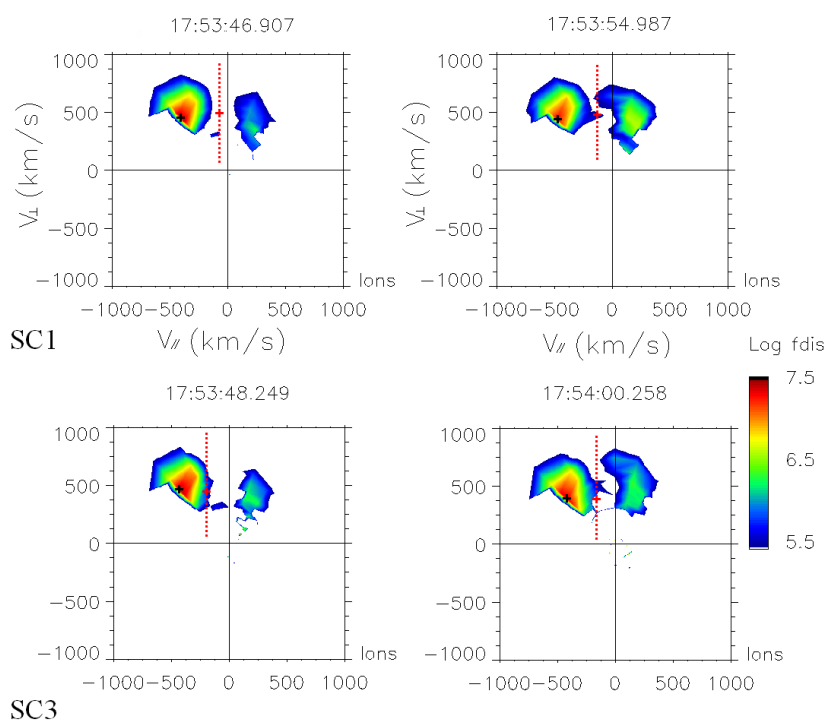

Fig. 6. 17 February 2004. Bi-dimensional cut-off of the ion distribution function in the Vper-Vpar plane for spacecraft 1 (top panels) and spacecraft 3 (bottom panel) from HIA instrument. The red cross represent the velocity of the deHoffmann-Teller frame and the black cross the velocity of the main ion population. Note that the ion distribution function of the transmitted ion population is truncated because of the operating mode of the HIA instrument.

only detected variation at that time is a decrease of the $V_{Y \mathrm{GSE}}$ velocity from about $100 \mathrm{~km} / \mathrm{s}$ to $0 \mathrm{~km} / \mathrm{s}$ with no notable modification of the other components. It is more difficult to estimate the error during the measurement of ion beams as the ion velocity is quickly changing. We used Whisper plasma frequency measurement to estimate this error. Unfortunately this indirect measurement of the plasma density is perturbed by the presence of large band emissions (P. Canu, private communication). This measurement has thus a big uncertainty. For example, with Whisper we obtain a density comprised between 1 and $1.6 \mathrm{~cm}^{-3}$ at 18:00 TU whereas the HIA instrument gives a density of $0.65 \mathrm{~cm}^{-3}$. It is thus difficult to obtain a precise corrective coefficient in the magnetopause boundary layer. However, the density underestimation can be roughly estimated as being close to two in this region too.

This underestimation of the density leads to an overestimation of the Alfvén velocity of a factor of about $\sqrt{ } 2$. Furthermore, the Alfvén velocity has been computed considering that the pressure anisotropy $\alpha$ was null because it is impossible to determine it accurately. Indeed, the inadequate operating mode of the CIS experiment leads to an incomplete sampling of the ion distribution function and causes errors in the computation of the perpendicular and parallel temperatures. This assumption can also affect in a lower degree the result of the Walén test.

After correcting the density, the ion velocity in the dHT frame is about 0.7 to $0.9 V_{A}$, which constitutes a satisfy- ing result for the Walén test (Khrabov et Sonnerup, 1998). Indeed, while the directional change of the plasma velocity is generally in good agreement with the Alfvén velocity, the magnitude of the velocity change is usually smaller than the Alfvén speed. The good correlation coefficient of the component-by-component scatter plot and the smaller than $V_{A}$ plasma velocity reveal a similar behaviour in this event. The cause of this discrepancy is not understood yet but several explanations have been proposed (Paschmann and Sonnerup, 2008). This includes among other things the underestimation of the density due to the presence of cold particle populations, two- or three-dimensional effects and nongyrotropic plasma behaviour.

The plasma flow in the dHT frame is oppositely directed to the magnetic field as evidenced by the negative sign of $p_{W a}$. It corresponds to a reconnection site located northward of the satellite, in agreement with the orientation of the magnetic field along the magnetopause normal.

\subsection{Ion distribution function}

The last step to reveal the reconnection process is to examine the ion distribution function. In particular, one of the most important kinetic signatures of the reconnection process is the presence of $\mathrm{D}$-shaped distribution functions as predicted by the reconnection theory. Indeed, in the dHT frame, only the particles with positive (northward of the reconnection site) or negative (southward of the reconnection site) velocities along the magnetic field can be transmitted through the magnetopause (Cowley et al., 1982). In a fixed frame, it induces a cutoff of the ion distribution function at $V_{H T}$ along the magnetic field and therefore the ion distribution function must exhibit a D-shape (e.g. Gosling et al., 1991; Fuselier et al., 2000; Phan et al., 1996).

Two consecutive slices of the ion distribution function in the $\left(V_{\mathrm{PAR}}-V_{\mathrm{PERP}}\right)$ frame obtained with the Cluster 1 and 3 HIA instruments for the accelerated flows at $\sim 17: 54$ UT are given in Fig. 6. These distribution functions, almost identical for the two spacecraft, show the presence of two ion populations.

The first one, with a negative velocity along the magnetic field, corresponds to the $\mathrm{H}^{+}$magnetosheath ions transmitted through the magnetopause. The distribution function is truncated in its lower part due to the inadequate operating mode of the HIA instrument. It distorts slightly the ion distribution function and makes difficult the identification of the predicted D-shape. However, we clearly see a cut-off of the parallel velocities at $V_{H T}$.

The second population is flowing in the magnetic field direction. It consists of magnetosheath ions, which have penetrated into the magnetosphere and then have been reflected by the magnetic mirror force at low altitude.

Both populations are flowing at $V_{H T \perp}$ in the direction perpendicular to the magnetic field. The existence of these two populations has been predicted by kinematic considerations 
Table 2. Walén test results for the two consecutive magnetopause crossings discussed in detail in this section. $p_{W a}$ represents the fitting slope of $\left(\mathbf{V}-\mathbf{V}_{H T}\right)$ versus $\mathbf{V}_{A}$ and $c_{W a}$ the corresponding correlation coefficient. The last column indicates the value of $p_{W a}$ after correction of the density. These results don't take into account the density correction due to the inadequate operating mode of the CIS experiment.

\begin{tabular}{ccccc}
\hline & & $\mathrm{pWa}$ & $\mathrm{cWa}$ & corrected pWa \\
\hline \multirow{2}{*}{ Satellite 1 } & $17: 53: 26-17: 54: 31$ (Msh-Msph) & -0.5926 & 0.9632 & $-0,8380$ \\
& $17: 54: 39-17: 55: 35$ (Msph-Msh) & -0.4849 & 0.9468 & -0.6857 \\
Satellite 3 & $17: 53: 18-17: 54: 30$ (Msh-Msph) & -0.5790 & 0.9614 & -0.8188 \\
& $17: 54: 30-17: 55: 42$ (Msph-Msh) & -0.4609 & 0.9576 & -0.6518 \\
\multirow{2}{*}{ Satellite 4 } & $17: 53: 22-17: 54: 34$ (Msh-Msph) & -0.5543 & 0.9359 & -0.7838 \\
& $17: 54: 42-17: 55: 38$ (Msph-Msh) & -0.6214 & 0.9708 & -0.8787 \\
\hline
\end{tabular}

\section{CIS-HIA SC $3 \quad 17 / \mathrm{Feb} / 2004 \quad 17: 53: 48.249$}
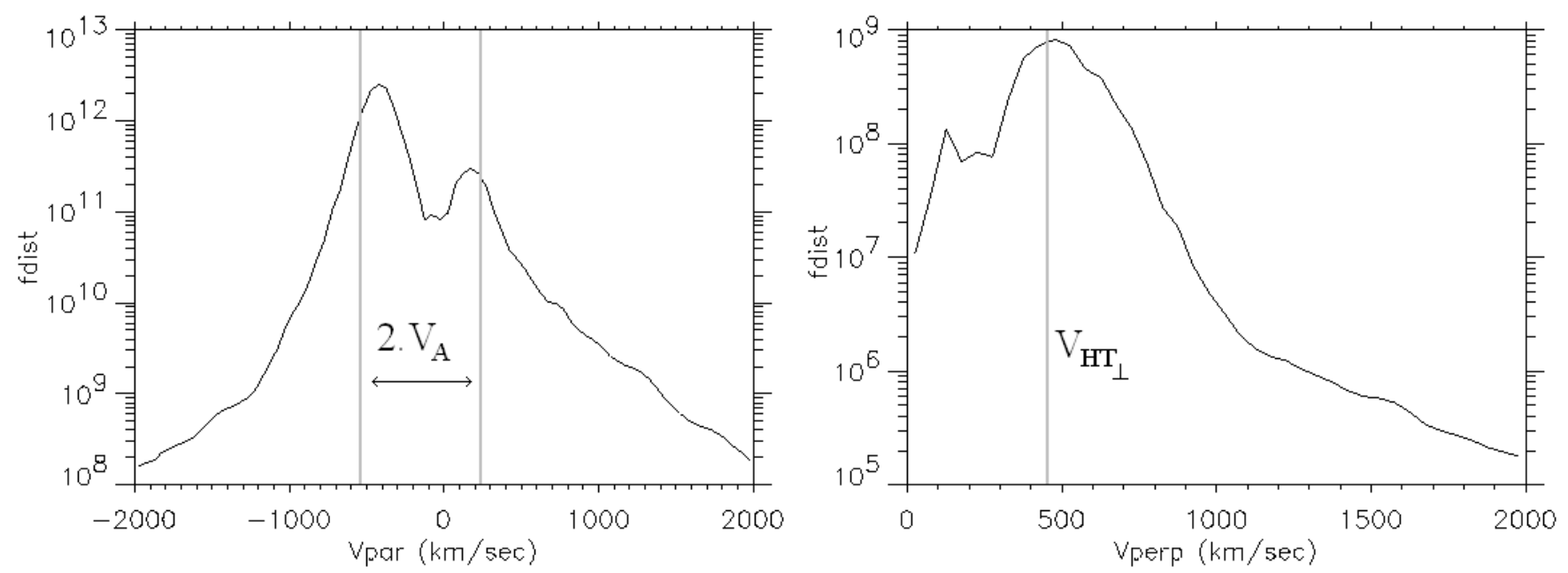

Fig. 7. 17 February 2004, 17:53:48.289 UT. Ion distribution function with respect to their velocity parallel to the magnetic field (left panel) and to their velocity perpendicular to the magnetic field (right panel) from SC3 HIA instrument. In the left panel, the two vertical lines represent respectively the component parallel to the magnetic field of $V_{H T}-V_{A}$ and $V_{H T}+V_{A}$. In the right panel the vertical line represents the component of the dHT velocity perpendicular to the magnetic field.

(Cowley, 1982) and observed, in particular at high latitude near the cusp region (e.g. Fuselier et al., 2000). However this is to our knowledge the first observation of a reflected ion population in the dayside magnetopause at such a high geocentric distance. Figure 7 displays the ion distribution function with respect to the parallel and perpendicular velocity measured by SC3 at 17:53:48 UT. In the direction perpendicular to the magnetic field, only one peak is observed at $V_{H T \perp}$. On the contrary, in the magnetic field direction, we clearly observe two peaks. The first one, at $V_{H T / /}-V_{A}$ corresponds to the transmitted ions and the second one at $V_{H T / /}+V_{A}$ corresponds to the reflected ions. According to the theory, the reflected and transmitted populations are flowing at respectively $+V_{A}$ and $-V_{A}$ in the $\mathrm{dHT}$ frame.

Previous studies reported the presence of low speed cutoffs of the transmitted and reflected populations. Interpreted as a velocity filter effect caused by the convection of the reconnected field line, they have been used to estimate the location of the X-line (e.g. Fuselier et al., 2000). In particular, a low speed cut off is expected to be observed on the mirrored population if the spacecraft is not too far from the reconnection region. Indeed, only particles with a sufficient parallel velocity have time to be mirrored and reach the spacecraft during the time the reconnected field line is convected from the X-line to the spacecraft. In this event, the absence of velocity cut off means that Cluster spacecraft are far from the reconnection site. Thus, we can not use this method to estimate the location of the X-line.

However, by simple considerations, we can nevertheless roughly estimate the location of the reconnection site. The magnetic field lines length between Cluster and the high altitude ionosphere is about $19 R_{E}$ in a simple dipolar magnetic 


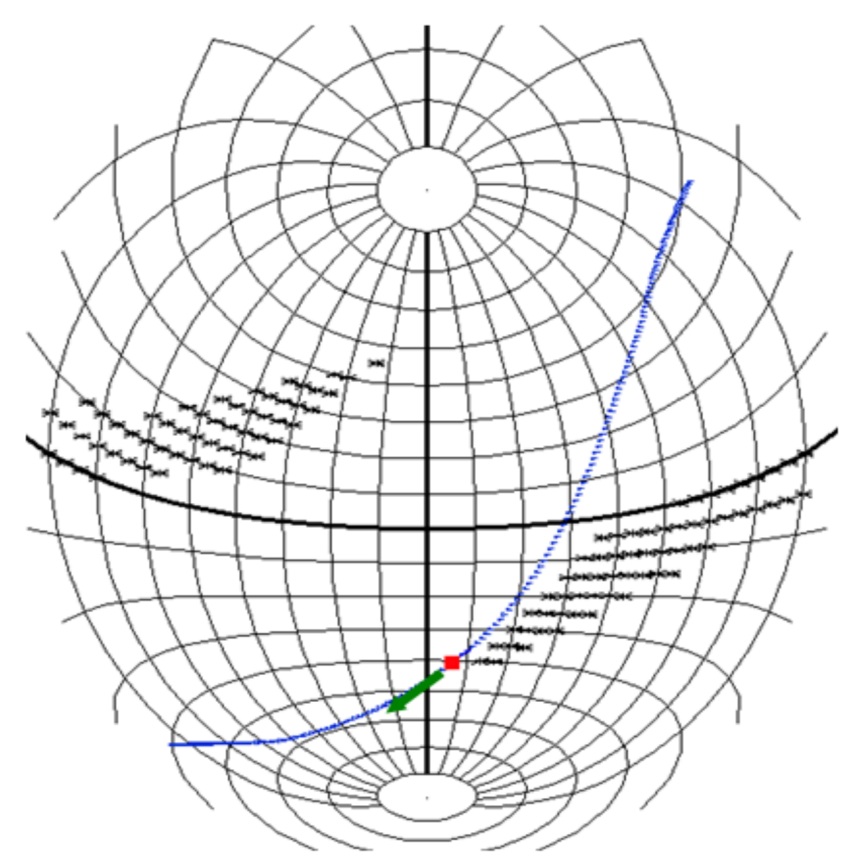

Fig. 8. Front view of the magnetopause. The red cross represents the location of Cluster spacecraft at 18:00 UT. The blue line represents the IMF line passing by Cluster spacecraft. The regions indicated by black crosses are regions of high magnetic shear where antiparallel reconnection models expect reconnection to occur. The green arrow indicated the direction of convection at Cluster location as obtained by the dHT analysis. The reconnection site is estimated to lie in a region located in the opposite direction of the convection velocity at some tens of $R_{E}$ from Cluster spacecraft.

field model. Supposing that the transmitted ions are reflected close to the earth, the distance they travelled to go from their entrance site in the magnetosphere to their mirror point and then to come back to Cluster spacecraft is of about $2 \times 19=38 R_{E}$. Because these ions travel along the magnetic field lines at the Alfvén velocity $(\sim 350-400 \mathrm{~km} / \mathrm{s})$, their travel time is estimated around $(38 \times 6378) / 400=600 \mathrm{~s}$ or $10 \mathrm{~min}$. During that time, the reconnected magnetic field lines are convected along the magnetopause. From the dHT frame velocity in the magnetopause plane (or equivalently from the ion perpendicular velocity) we estimate the convection velocity to be approximately $400 \mathrm{~km} / \mathrm{s}$. Therefore, the reconnection site may be located at a distance higher than $600 \times 400=240000 \mathrm{~km}$ or $38 R_{E}$ from the observation point.

Obviously, this is a rough estimation of the location of the $\mathrm{X}$-line. However, we can state that the observation of a reflected population at such a high geocentric distance implies that the Cluster spacecraft are located at several tens of $R_{E}$ from the merging region. This very high distance has to be considered taking into account the large size of the magnetosphere during this time period. Furthermore, according to the direction of the dHT velocity (on average in the GSM frame: $\left.V_{H T X}=-188 \mathrm{~km} / \mathrm{s}, V_{H T Y}=-273 \mathrm{~km} / \mathrm{s}, V_{H T Z}=-269 \mathrm{~km} / \mathrm{s}\right)$, we estimate that the reconnection site may be located in the duskward side of the dayside magnetopause. As shown by a mapping of IMF magnetic field line on the magnetopause, the magnetic shear across the magnetopause is high in this region. This seems consistent with antiparallel reconnection model predictions of the X-line location for the corresponding IMF conditions (Fig. 8). However, due to low precision in the determination of the location of the X-line, we cannot claim that the antiparallel reconnection model better describes the large scale configuration of magnetic reconnection than component reconnection during this event.

Another information is deduced from the presence of the reflected population. The reflected ions are detected during every magnetopause crossing, in particular during the first one at around 17:50 UT (not shown). As a consequence, the reconnection process must have started at least $10 \mathrm{~min}$ before 17:50 UT, the time for magnetosheath ions to enter the magnetosphere, be reflected and reach the Cluster spacecraft. We conclude that the reconnection onset must have occurred before 17:40 UT.

\section{Magnetopause motion and thickness}

Finally, we turn to an evaluation of the motion and thickness of the magnetopause with two different methods (Table 3).

We first made a multi-spacecraft analysis to estimate the magnetopause motion along its normal. We use the time delays for the magnetopause crossings between different Cluster spacecraft obtained with high-resolution magnetic field data $(5 \mathrm{~Hz})$. The magnetopause is assumed to be a planar surface, which may be a valuable assumption due to the low separation distance between Cluster spacecraft during this event. From the plasma and magnetic field measurements, we expect an inward motion for the first magnetopause crossing and an outward motion for the second one. We obtain a magnetopause velocity comprised between $-19.5 \mathrm{~km} / \mathrm{s}$ and $-26.4 \mathrm{~km} / \mathrm{s}$ and between $17.5 \mathrm{~km} / \mathrm{s}$ and $22.3 \mathrm{~km} / \mathrm{s}$, respectively, for the inward and outward motion. Note that we did not use the Cluster 2 spacecraft as its separation distance from the Cluster 3 spacecraft along the magnetopause normal is too short.

The second method consists of a single spacecraft analysis. Here, the magnetopause motion along its normal is evaluated by projecting the $\mathrm{dHT}$ velocity along the magnetopause normal. The velocities are comprised between $-44.5 \mathrm{~km} / \mathrm{s}$ and $-69.4 \mathrm{~km} / \mathrm{s}$ and between $-1.6 \mathrm{~km} / \mathrm{s}$ and $23.8 \mathrm{~km} / \mathrm{s}$, respectively, for the first and second crossing. This method gives more variable results because of its high sensitivity to the magnetopause normal orientation (Khrabov and Sonnerup, 1998) and must be less reliable than the time delay analysis.

In the following, we will use the velocity obtained by the first method and the measured duration of the crossings to estimate the magnetopause thickness. 
For the first magnetopause crossing, from the magnetosphere to the magnetosheath, the magnetic fields measured by SC3 and SC4 at the beginning and at the end of the crossing are similar while they are separated in the direction normal to the magnetopause. It indicates that these two satellites are located in regions where the magnetic field is almost uniform and thus that they have crossed the magnetopause current layer almost entirely. On the contrary, the magnetic field variations detected by SC1 are less pronounced than those detected by the other Cluster spacecraft. This spacecraft, which is the closest to the earth, has only partially crossed the current sheet and will not be used to estimate its thickness.

The magnetic field rotation during this crossing lasts $35 \mathrm{~s}$ for SC3 and $40 \mathrm{~s}$ for SC4. The magnetopause thickness is thus estimated between $23 \times 35 \sim 800 \mathrm{~km}$ and $23 \times 40 \sim 900 \mathrm{~km}$. For the second crossing, the magnetic field fluctuations (in particular in the $Y_{\mathrm{GSE}}$ direction) make difficult this kind of analysis.

We made a similar analysis for the other magnetopause crossings and obtained comparable results. These values are compatible with those obtained during standard solar wind conditions with ISEE spacecraft at similar latitudes: 10$80 \mathrm{~km} / \mathrm{s}$ for the magnetopause velocity and 400-1000 km for the magnetopause thickness (Berchem and Russel, 1982). However we would expect a thicker magnetopause during this event as the ion gyroradii is about three times higher than during nominal solar wind conditions due to the low magnetic field value. Indeed, the magnetopause thickness, corresponding to 3 protons gyroradii is lower than the average value of 10 gyroradii obtained by Berchem and Russell (1982) albeit it is in the range of values obtained in their statistical study.

\section{Conclusion}

We have presented in-situ measurements taken in the magnetosheath/magnetopause during exceptional conditions when the solar wind almost disappeared. Due to the low solar wind density, the magnetosheath beta was very low $(0.15)$ and the magnetosphere was dilated and extended up to $16 R_{E}$ in the solar direction.

Between 17:50 UT and 18:10 UT Cluster spacecraft cross several times the magnetopause and each of these crossings is associated with the presence of high velocity plasma jets.

We showed the presence of a magnetic field of a few nT along the magnetopause normal. We also evidenced that the tangential stress balance was satisfied. The analysis of the ion distribution function revealed the presence of D-shaped ion distribution functions and of a reflected ion population. Both fluid and kinetic considerations are consistent and evidence that the high velocity plasma jets result from reconnection in the dayside magnetopause.
Table 3. Magnetopause velocity in $\mathrm{km} / \mathrm{s}$ deduced from the time delays between different spacecraft crossings and from the projection of the dHT velocity along the magnetopause normal.

\begin{tabular}{lccc}
\hline & Satellites & Msh-Msph & Msph-Msh \\
\hline \multirow{3}{*}{ Multi spacecraft analysis } & $1-3$ & -26.4 & 22.3 \\
& $1-4$ & -23.2 & 20.1 \\
& $3-4$ & -19.5 & 17.5 \\
\hline \multirow{3}{*}{$\boldsymbol{V} \boldsymbol{n} \boldsymbol{n}$} & 1 & -44.5 & -1.6 \\
& 3 & -51.3 & 23.8 \\
& 4 & -69.4 & 15.2 \\
\hline
\end{tabular}

Inside the plasma jets, the Cluster spacecraft simultaneously detect reflected ions. This is the first time to our knowledge that reflected ions are observed at such a high geocentric distance. Because of this large distance, the time of flight of these ions is important (about $10 \mathrm{~min}$ ). It implies that the reconnection site is located at several tens of Earth radii from Cluster spacecraft. The direction of the magnetic field lines convection at the Cluster location suggests that the reconnection site is probably located in the dusk side of the dayside magnetopause.

This reflected population also indicates that reconnection started $10 \mathrm{~min}$ before the Cluster spacecraft detect the accelerated plasma jets (from about 17:50 UT to 18:10 UT). We interpret it as an evidence for continuous reconnection during more than half an hour. However, we cannot totally exclude that the reconnection process ceases between the jets when the Cluster spacecraft are located in the magnetosheath but the systematic observation of plasma jets at each magnetopause crossing strongly indicates continuous reconnection.

Cluster magnetic field measurements reveal that a southward turning of the IMF reached the Cluster spacecraft between 17:50 UT and 18:10 UT. However, it may have reached other regions of the magnetopause before that time. Because the $\mathrm{X}$ component of the IMF is low, the southward turning must have reached the subsolar point before the Cluster spacecraft. The separation distance between the subsolar point and the Cluster spacecraft along the $X_{\mathrm{GSE}}$ axis is of about $16-12.5=3.5 R_{E}$. Taking a propagation speed into the magnetosheath of $1 / 8$ of the solar wind velocity (Spreiter and Stahara, 1980), the IMF would reach the subsolar point $(3.5 \times 6378) /(450 / 8) \sim 400 \mathrm{~s}$ or $\sim 6.5 \mathrm{~min}$ before the Cluster spacecraft if it had a purely southward orientation. This delay is a bit short compared to the estimated time of the reconnection onset but it doesn't take into account neither the actual orientation of the discontinuity associated with the IMF southward turning nor the azimuthal solar wind velocity and the possible associated magnetopause distortion. However, it implies that if this IMF reversal initiated the dayside reconnection process then the reconnection onset must have been very fast. 
These observations tend to show that during this period of low solar wind density, magnetic reconnection was efficient and provided solar wind plasma to the magnetosphere. This is consistent with statistical study of accelerated flows at the magnetopause (Scurry et al., 1994a, b) which revealed that the merging process depends on the local magnetosheath beta. According to this study, the lower is the magnetosheath beta, the higher is the probability to observe accelerated flows at the magnetopause.

Furthermore, when the solar wind dynamic pressure is very low as during this event, the magnetosphere is extremely dilated and its cross section with the solar wind is high. The size of the region where solar wind plasma penetrates into the magnetosphere via magnetic reconnection must therefore be more extended than during standard conditions.

Because of its possible increased efficiency and because of the dilatation of the magnetosphere, magnetic reconnection may be more efficient to provide solar wind plasma to the magnetosphere during periods of low solar wind density. It can partially compensate the low density of the solar wind source. This could explain why during prolonged (a few days) periods of low solar wind density, the plasmasheet and magnetotail are maintained (Terasawa et al., 2000).

Finally, we also showed that the magnetopause thickness $(\sim 800 \mathrm{~km})$ and velocity $(\sim 20 \mathrm{~km} / \mathrm{s})$ do not differ from the average values given by statistical studies (e.g. Berchem and Russell, 1982) and that, while the magnetosphere is extremely extended, its location is still well predicted by the Shue et al. (1997) magnetopause model.

Acknowledgements. We thank the principal investigators of ACE MAG and SWEPAM experiments for making their data avaible.

The authors are grateful to E. Penou for the development of the Cluster CIS software. R. Maggiolo thanks M. Roth for helpful discussion and comments.

Topical Editor I. A. Daglis thanks J. De Keyser and A. V. Dmitriev for their help in evaluating this paper.

\section{References}

Arnoldy, R. L.: Signature in the interplanetary medium for substorms, J. Geophys. Res., 76, 5189-5201, 1971.

Balogh, A., Carr, C. M., Acuña, M. H., Dunlop, M. W., Beek, T. J., Brown, P., Fornaçon, K.-H., Georgescu, E., Glassmeier, K.H., Harris, J., Musmann, G., Oddy, T., and Schwingenschuh, K.: The Cluster Magnetic Field Investigation: overview of in-flight performance and initial results, Ann. Geophys., 19, 1207-1217, 2001, http://www.ann-geophys.net/19/1207/2001/.

Bavassano Cattaneo, M. B., Marcucci, M. F., Retinò, A., Pallocchia, G., Rème, H., Dandouras, I., Kistler, L. M., Klecker, B., Carlson, C. W., Korth, A., McCarthy, M., Lundin, R., and Balogh, A.: Kinetic signatures during a quasi-continuous lobe reconnection event: Cluster Ion Spectrometer (CIS) observations, J. Geophys. Res., 111, 9212, doi:10.1029/2006JA011623, 2006.

Berchem, J. and Russell, C. T.: The thickness of the magnetopause current layer - ISEE 1 and 2 observations, J. Geophys. Res., 87, 2108-2114, 1982.
Bosqued, J. M., Phan, T. D., Dandouras, I., Escoubet, C. P., Rème, H., Balogh, A., Dunlop, M. W., Alcaydé, D., Amata, E., Bavassano-Cattaneo, M.-B., Bruno, R., Carlson, C., DiLellis, A. M., Eliasson, L., Formisano, V., Kistler, L. M., Klecker, B., Korth, A., Kucharek, H., Lundin, R., McCarthy, M., McFadden, J. P., Möbius, E., Parks, G. K., and Sauvaud, J.-A.: Cluster observations of the high-latitude magnetopause and cusp: initial results from the CIS ion instruments, Ann. Geophys., 19, 1545-1566, 2001, http://www.ann-geophys.net/19/1545/2001/.

Cowley, S. W. H.: The causes of convection in the earth's magnetosphere -A review of developments during the IMS, Rev. Geophys. Space Phys., 20, 531-565, 1982.

Escoubet, C. P., Fehringer, M., and Goldstein, M.: The Cluster mission, Ann. Geophys., 19, 1197-1200, 2001, http://www.ann-geophys.net/19/1197/2001/.

Fuselier, S. A., Trattner, K. J., and Petrinec, S. M.: Cusp observation of high- and low-latitude reconnection for northward interplanetary magnetic field, J. Geophys. Res., 105, 253-266, 2000.

Gosling, J. T., Thomsen, M. F., Bame, S. J., Elphic, R. C., and Russell, C. T.: Observations of reconnection of interplanetary and lobe magnetic field lines at the high-latitude magnetopause, J. Geophys. Res., 96, 14 097-14 106, 1991.

Hudson, P. D.: Discontinuities in an anisotropic plasma and their identification in the solar wind, Planet. Space Sci., 18, 16111622, 1970.

Janardhan, P., Fujiki, K., Sawant, H. S., Kojima, M., Hakamada, K., and Krishnan, R.: Source regions of solar wind disappearance events, J. Geophys. Res., 113, 3102-3110, 2008.

Khrabrov, A. V. and Sonnerup, B. O.: DeHoffmann-Teller analysis. Analysis Methods for Multi-Spacecraft Data, ISSI Scientific Report SR-001, ESA Pub. Div., 221, 221-248, 1998.

Lockwood, M.: Astronomy: the day the solar wind nearly died, Nature, 409(6821), 677-679, 2001.

Parks, G., Brittnacher, M., Chua, D., Fillingim, M., Germany, G., and Spann, J.: Behaviour of the aurora during 10-12 May, 1999 when the solar wind nearly disappeared, Geophys. Res. Lett., 27, 4033-4036, 2000.

Paschmann, G., Sonnerup, B. U. O., Papamastorakis, I., Sckopke, N., Haerendel, G., Bame, S. J., Asbridge, J. R., Gosling, J. T., Russell, C. T., and Elphic, R. C.: Plasma acceleration at the Earth's magnetopause, Evidence for magnetic reconnection, Nature, 282, 243-246, 1979.

Paschmann, G. and Sonnerup, B. O.: DeHoffmann-Teller analysis. Analysis Methods for Multi-Spacecraft Data, ISSI Scientific Report SR-008, ESA Pub. Div., 65, 65-74, 2008.

Phan, T. D., Paschmann, G., and Sonnerup, B. U. O.: Low-latitude dayside magnetopause and boundary layer for high magnetic shear 2. Occurrence of magnetic reconnection, J. Geophys. Res., 101, 7871-7828, 1996.

Rème, H., Aoustin, C., Bosqued, J. M., et al.: First multispacecraft ion measurements in and near the earth's magnetosphere with the identical Cluster Ion Spectrometry (CIS) Experiment, Ann. Geophys., 19, 1303-1354, 2001, http://www.ann-geophys.net/19/1303/2001/.

Scurry, L., Russell, C. T., and Gosling, J. T.: Geomagnetic activity and beta dependence of the dayside reconnection rate, J. Geophys. Res., 99, 14 811-14814, 1994a.

Scurry, L., Russell, C. T., and Gosling, J. T.: A statistical study of accelerated flow events at the dayside magnetopause, J. Geophys. 
Res., 99, 14 815-14 829, 1994b.

Shue, J.-H., Chao, J. K., Fu, H. C., Russell, C. T., Song, P., Khurana, K. K., and Singer, H. J.: A new functional form to study the solar wind control of the magnetopause size and shape, J. Geophys. Res., 102, 9497-9512, 1997.

Sibeck, D. G., Lopez, R. E., and Roelof, E. C.: Solar wind control of the magnetopause shape, location and motion, J. Geophys. Res., 96, 5489-5495, 1991.

Sonnerup, B. U. O., Paschmann, G., Papamastorakis, I., Sckopke, N., Haerendel, G., Bame, S. J., Asbridge, J. R., Gosling, J. T., and Russell, C. T.: Evidence for magnetic reconnection at the Earth's magnetopause, J. Geophys. Res., 86, 10 049-10 067, 1981.
Spreiter, J. R. and Stahara, S. S.: A new predictive model for determining solar wind-terrestrial planet interactions, J. Geophys. Res., 85, 6769-6777, 1980.

Tenuous solar wind: Special issue, Geophys. Res. Lett., 27, 37613792, 2000.

Terasawa, T., Kasaba, Y., Tsubouchi, K., Mukai, T., Saito, Y., Frank, L. A., Paterson, W. R., Ackerson, K., Matsumoto, H., Kojima, H., Matsui, H., Larson, D., Lin, R., Phan, T., Steinberg, J., McComas, D., Skoug, R., Fujimoto, M., Hoshino, M., and Nishida, A.: GEOTAIL observations of anomalously low density plasma in the magnetosheath, Geophys. Res. Lett., 27(23), 3781-3784, 2000. 